\title{
NOUVELLE
}

\section{Un mécanisme inédit de détournement viro-induit de 53 dans le contexte de l'infection par les virus influenza}

Julia Dubois, Manuel Rosa-Calatrava, Olivier Terrier
Centre international de recherche en infectiologie (équipe VirPath), Univ Lyon, Inserm U1111, Université Claude Bernard Lyon 1, CNRS UMR5308, École normale supérieure de Lyon, F-69007 Lyon, France. olivier.terrier@univ-lyonl.fr
Rôle central joué par la protéine virale NS1 dans l'infection par les virus influenza

L'infection par les virus influenza, agents de la grippe, constitue un problème majeur de santé publique, avec notamment un impact important sur les populations à risque lors des épidémies saisonnières. De nombreuses équipes de recherche, dont la nôtre, s'intéressent aux interactions entre ces virus et la cellule hôte afin de mieux comprendre les mécanismes de l'infection et proposer de nouvelles stratégies thérapeutiques. Contrairement à la plupart des autres virus à $A R N$, une partie du cycle viral des virus influenza s'accomplit dans le compartiment nucléaire de la cellule hôte, ce qui implique des interactions avec de nombreux facteurs cellulaires au profit de la réplication virale. Les virus influenza ont notamment développé des stratégies pour détourner la machinerie cellulaire d'épissage des transcrits primaires, afin, d'une part, d'altérer la réponse de la cellule hôte à l'infection, et, d'autre part, de permettre une expression optimale de certaines protéines virales [1]. La protéine virale non-structurale NSl est un véritable « couteau suisse » permettant au virus de moduler les réponses de l'hôte à l'infection, notamment la réponse interféron. Ce rôle central de NSl dans les interactions entre virus et cellule hôte s'exerce dans plusieurs mécanismes cellulaires comme l'épissage, la maturation des ARNm, le transfert nucléo-cytoplasmique des ARN, ou encore la traduction des ARNm, grâce à la capacité de NSI d'interagir avec de très nombreux partenaires cellulaires (plus de cinquante) par ses domaines de liaison à l'ARN double-brin et aux protéines p85 $\beta$, RIG-I, ou CPSF4 [2].

CPSF4 est un composant du complexe CPSF (cleavage and polyadenylation specificity factor), impliqué dans la dernière étape de maturation et de poly-adénylation des ARNm, mais aussi dans le contrôle de leur épissage alternatif. Dans le cas de l'infection par les virus influenza, il a été montré que la liaison de NSI à CPSF4 perturbe sa liaison aux pré-ARNm de la cellule hôte et inhibe leur clivage à l'extrémité 3 ' et leur poly-adénylation. Cette interaction contribue ainsi au contrôle NS1dépendant de l'expression des gènes de l'hôte, se traduisant en particulier par l'inhibition de la réponse interféron [3]. La liaison de NS1 à CPSF4 pourrait également affecter plus spécifiquement l'expression de certains gènes de l'hôte en perturbant l'épissage alternatif de leurs ARNm.

Interactions fonctionnelles entre virus influenza et le facteur de transcription p53

La protéine régulatrice $p 53$ est impliquée dans un grand nombre de phénomènes cellulaires, comme le cycle cellulaire, l'apoptose ou la sénescence, ainsi que dans la réponse immune et inflammatoire. Ce facteur de transcription impliqué dans l'homéostasie cellulaire, et dont on a célébré récemment les 40 ans de la découverte, a surtout été étudié en oncologie. Cependant, nous savons que la protéine p53 est impliquée dans le contrôle des infections virales au sens large, tous les virus, oncogènes ou non, ayant développé une palette de stratégies différentes pour moduler ou détourner ses fonctions afin d'optimiser leur réplication dans la cellule hôte [4].

Dans le cas des virus influenza, les travaux menés par notre équipe ont permis de montrer qu'il existe de multiples interactions fonctionnelles entre ces virus et $p 53$. Les virus influenza sont ainsi capables de moduler l'expression endogène et l'activité transcriptionnelle de p53 au cours de l'infection, contribuant à maintenir un état cellulaire favorable à la réplication virale [5-7]. Dans ce modèle d'infection, la protéine multifonctionnelle NSl semble jouer un rôle majeur. Nous avons en effet montré que NSl interagit directement avec p53, et que cette interaction perturbe la liaison du facteur de transcription aux régions promotrices de ses gènes cibles [5-7]. Cependant, le mécanisme de la modulation de l'activité de p53 par NS1 n'était pas encore entièrement compris.

\section{L'infection par les virus influenza} modifie l'épissage du transcrit de TP53 En plus de la forme canonique de p53 (aussi appelée $p 53 \alpha$ ), le gène TP53 code 12 isoformes distinctes grâce à la présence de différents promoteurs, sites 


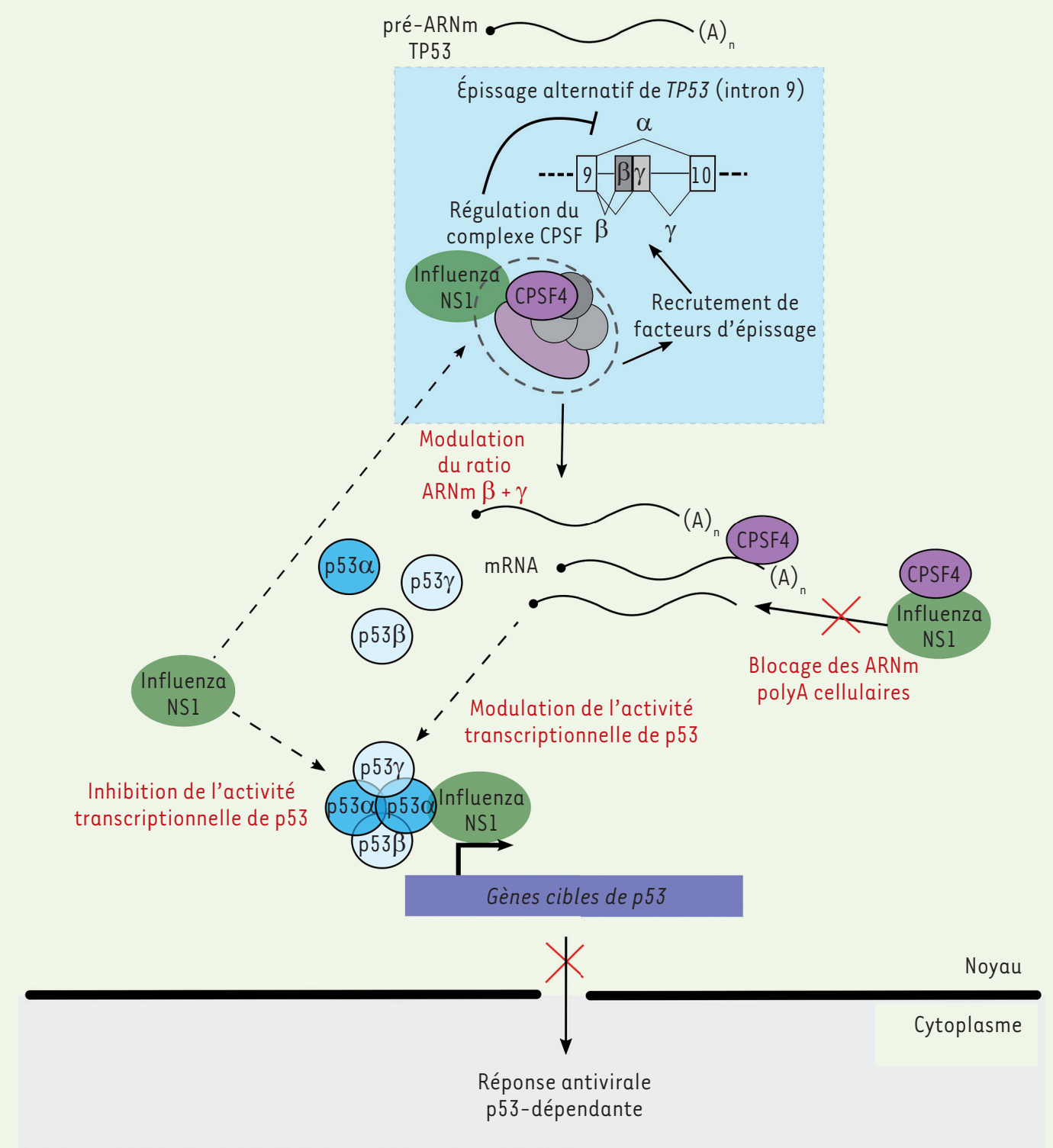

Figure 1. Modèle rendant compte de l'interaction fonctionnelle entre protéine virale NS1, épissage du transcrit de TP53, et CPSF4. Lors de l'infection, la protéine virale NSI inhibe l'activité transcriptionnelle de p53 en se liant à cette protéine, mais également en modulant l'épissage du préARNm de TP53, et en affectant la fonction du facteur cellulaire CPSF4 dans l'épissage et la maturation des ARNm. En conséquence, la modulation de la proportion relative des isoformes de 533 a un impact sur l'activité transcriptionnelle de $p 53$, notamment sur la régulation de nombreux gènes impliqués dans la réponse antivirale.

d'épissage, et sites d'initiation de la traduction. Ces isoformes sont impliquées dans la régulation de $\mathrm{p} 53 \alpha$ et les réponses biologiques qui en dépendent. Nous avons montré que l'infection par les virus influenza modifiait considérablement l'expression de ces isoformes, dont celles issues d'épissages différents du transcrit primaire ( $p 53 \beta$ et $p 53 \gamma)$, et que cette modulation avait en retour un impact important sur la réplication virale, ce qui révélait un niveau supplémentaire de complexité dans les interactions entre virus influenza et p53 [8]. Plus récemment, afin de mieux comprendre l'impact de l'infection sur l'épissage du transcrit de TP53, nous avons utilisé une approche fondée sur
I'utilisation d'un minigène permettant d'étudier de manière spécifique l'exclusion ou la rétention partielle de l'intron 9 du transcrit de TP53, à l'origine des formes $\alpha, \beta$ et $\gamma$ [9]. Nous avons ainsi pu montrer que l'infection favorisait la rétention de l'intron 9, donc les isoformes $\beta$ et $\gamma$ de la protéine, conformément à nos observations précédentes. 
Interaction fonctionnelle entre NS1, CPSF4, et l'épissage du transcrit de TP53, et son impact sur la réponse interféron de type I p53-dépendante Compte tenu de la contribution de la protéine virale NSl dans la modulation de la machinerie d'épissage, nous avons recherché si NSI jouait un rôle dans la rétention de l'intron 9 dans le transcrit de TP53. En utilisant un minigène, nous avons montré que l'expression transitoire de NSI avait le même effet sur la rétention de l'intron 9 que I'infection virale. Cet effet n'était plus observé en présence d'une protéine NSl porteuse de mutations supprimant son interaction avec le facteur CPSF4 [9]. Différentes expériences utilisant des petits ARN inhibiteurs (si-RNA) de l'expression endogène de CPSF4 ou de l'élimination de l'intron 9 au cours de l'épissage du transcrit de TP53, mais aussi des virus influenza recombinants dont la protéine NSI mutée est incapable d'interagir avec CPSF4, nous ont ensuite permis de préciser l'interaction fonctionnelle entre NS1, CPSF4, l'épissage du transcrit de TP53, et l'activité transcriptionnelle de la protéine p53. Ces expériences nous ont notamment permis de mettre en évidence le rôle du facteur CPSF4 dans l'épissage du transcrit de TP53, un rôle qui peut être modifié par l'interaction entre CPSF4 et NSI lors de l'infection virale. La modulation de l'épissage alternatif de ce transcrit se traduit par une modification des quantités relatives des isoformes $\alpha, \beta$ et $\gamma$ de la protéine p53, donc de l'activité transcriptionnelle de cette protéine, que nous avons pu observer pour plusieurs gènes cibles, dont MDM2 (Murine double minute 2), $B A X$ (BCl-2-associated $X$ ), et CDKNIA (Cyclin-dependent kinase inhibitor IA/ p21). Par ailleurs, le ciblage de l'expression des isoformes de p53 issues de l'épissage alternatif du transcrit à l'aide de petits ARN inhibiteurs spécifiques se traduit par une forte diminution de la production du virus influenza, ce qui plaide pour un rôle de p53 $\beta$ et p53 $\gamma$ favorisant la réplication virale, en accord avec nos observations précédentes. Nous avons également montré, dans le contexte de l'infection virale, que le facteur CPSF4 joue un rôle antiviral dépendant de $\mathrm{p} 53$, et que les isoformes $p 53 \beta$ et $p 53 \gamma$ jouent un rôle majeur dans le contrôle de la réponse interféron de type I passant par p53, CPSF4 étant vraisemblablement impliqué dans cette boucle de régulation [9]. Un schéma synthétisant l'interaction fonctionnelle complexe entre la protéine virale NSl et les protéines cellulaires CPSF4 et p53 est présenté dans la Figure 1. Nos résultats indiquent que le rôle des différentes isoformes de p53, en particulier celles issues de l'épissage alternatif du transcrit, dans le contrôle des infections virales a jusqu'à présent été sous-estimé [10]. $\diamond$

A novel mechanism for virally-induced hijacking of p53 identified in the context of influenza virus infection

\section{REMERCIEMENTS}

Les travaux de l'équipe ont bénéficié du soutien de l'université Claude Bernard Lyon 1. Julia Dubois a bénéficié d'une bourse de la région AuvergneRhône-Alpes (CMIRA ExploRA'DOC) et du soutien du consulat général de France à Québec (Programme Frontenac).

\section{LIENS D'INTÉRÊT}

Les auteurs déclarent n'avoir aucun lien d'intérêt concernant les données publiées dans cet article.

\section{RéFÉRENCES}

1. Dubois J, Terrier 0, Rosa-Calatrava M. Influenza viruses and mRNA splicing: doing more with less. MBio 2014 ; 5 : e 00070-14.

2. Marc D. Influenza virus non-structural protein NS1: interferon antagonism and beyond. J Gen Virol 2014 ; $95:$ 2594-611.

3. Nemeroff ME, Barabino SM, Li Y, et al. Influenza virus NS1 protein interacts with the cellular $30 \mathrm{kDa}$ subunit of CPSF and inhibits 3' end formation of cellular premRNAs. Mol Cell $1998 ; 1:$ :991-1000.

4. Aloni-Grinstein R, Charni-Natan M, Solomon H, Rotter V. p53 and the viral connection: back into the future. Cancers (Basel) 2018; 10 : pii : ع178.

5. Terrier 0, Josset L, Textoris J, et al. Cellula transcriptional profiling in human lung epithelial cells infected by different subtypes of influenza $A$ viruses reveals an overall down-regulation of the host $p 53$ pathway. Virol J $2011 ; 8: 285$

6. Terrier 0, Diederichs A, Dubois J, et al. Influenza NS1 interacts with $p 53$ and alters its binding to $p 53-$ responsive genes, in a promoter-dependent manner. FEBS Lett 2013 ; 587 : 2965-71.

7. Pizzorno A, Dubois J, Machado D, et al. Influenza A viruses alter the stability and antiviral contribution of host \&3-ubiquitin ligase Mdm2 during the time-course of infection. Sci Rep $2018 ; 8: 3746$.

8. Terrier 0 , Marcel V, Cartet $G$, et al. Influenza A viruses control expression of proviral human $\mathrm{p} 53$ isoforms p53 $\beta$ and $\Delta 133 p 53 \alpha$.J Virol $2012 ; 86: 8452-60$.

9. Dubois J, Traversier A, Julien T, et al. The nonstructural NS1 protein of influenza viruses modulates TP53 splicing through host factor CPSF4. J Virol 2019 ; 93 : pii : e02168-18.

10. Terrier 0, Bourdon JC, Rosa-Calatrava M. p53 protein isoforms: key regulators in the front line of pathogen infections? PLoS Pathog 2013 ; 9 : el 1003246.

\section{LA FONDATION PREMUP : UN OPÉRATEUR DE TERRAIN EN PÉRINATALITÉ RECONNU POUR SON EXCELLENCE ET SON INTERDISCIPLINARITÉ}

La Fondation de coopération scientifique PremUp, unique en Europe, intervient sur la prévention du handicap à la naissance, par la protection de la santé de la femme enceinte et du nouveau-né.

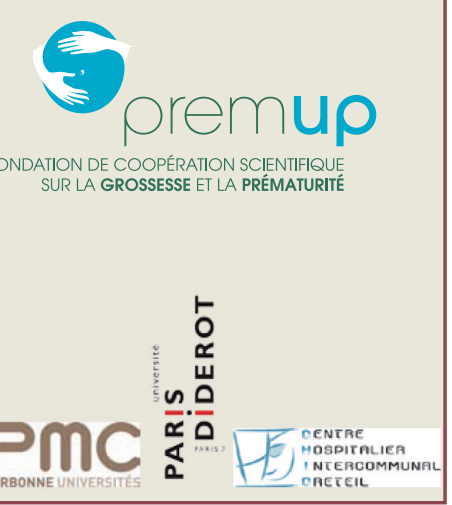

